\title{
Factors Affecting Phenotype Variability in a Family with CMT2B: Gender and LRSAM1 Genotype
}

\author{
Leema Reddy Peddareddygari $^{\mathrm{a}} \quad$ Kinsi Oberoi $^{\mathrm{a}} \quad$ Jaasrini Reddy Vellore ${ }^{\mathrm{a}}$ \\ Raji P. Grewal ${ }^{\text {b }}$ \\ ${ }^{a}$ The Neuro Genetics Institute, Sharon Hill, Pa., USA; ${ }^{b}$ Neuroscience Institute, Saint Francis \\ Medical Center, Trenton, N.J., USA
}

\section{Keywords}

Charcot-Marie-Tooth disease type 2B - Phenotype variability - Digeneic effect .

RABTA/LRSAM1 gene interactions

\begin{abstract}
Charcot-Marie-Tooth disease type 2 (CMT2) is an autosomal dominant axonal neuropathy caused by mutations in various genes. The subtype $C M T 2 B$ results from missense mutations in RABTA, member RAS oncogene family gene, whereas missense mutations in the Leucinerich repeat and sterile alpha motif-containing protein 1 (LRSAM1) gene cause CMT2P. We describe the genotype/phenotype analysis of a family in which a previously described mutation in the RABTA gene and a novel mutation in the LRSAM1 gene were identified. In this family, none of the individuals had ulceromutilating features, and there was a marked variability in the age of onset. We discuss the possible etiology of the observed phenotypic variability including the role of gender and possible RABTA/LRSAM1 gene interactions.
\end{abstract}




\section{Case Reports in Neurology}

\section{Introduction}

Charcot-Marie-Tooth disease (CMT) is one of the most common causes of peripheral neuropathy affecting at least 1 in every 2,500 people [1]. CMT is caused by mutations in a number of genes and can follow an autosomal dominant (AD), autosomal recessive (AR) or sex-linked pattern of inheritance. Type 2 CMT (CMT2) is an autosomal dominant neuropathy which is characterized electrophysiologically by axonal rather than by demyelinating features. The subsets of this disorder are further classified by the specific causative gene.

We present a patient who inherited mutations in RAB7A, member RAS oncogene family gene, and the Leucine-rich repeat and sterile alpha motif-containing protein 1 (LRSAM1) gene. Both of these genes are reported to cause different subtypes of CMT2 [2,3]. We propose that in this family, the disease phenotype may be have been modified by gender as well as by an interaction between these two genes.

\section{Methods}

\section{Clinical Feature}

The proband is a 36-year-old male who presented for evaluation with a history of progressive weakness of the distal arm and leg. He recalls that he first noted a clumsy gait beginning in his late teens. Since then, his symptoms have progressed and include numbness and tingling in a glove and stocking distribution, more marked in his legs. In his late twenties, he developed weakness and numbness of his hands. The weakness in his legs progressed to bilateral foot drop, and he currently requires bilateral ankle-foot orthosis to ambulate. There are no symptoms of cognitive difficulties, diplopia, dysphagia, tremor, seizures, pain or skin ulcerations.

Neurological examination revealed a normal mental status, and cranial nerve examination was also normal. Power testing showed decreased strength and atrophy of the distal muscles of the hand, and Medical Research Council (MRC) testing showed grade 3/5 abductor digiti minimi (ADM), first dorsal interossei, abductor pollicis brevis (APB) and MRC grade $4 / 5$ wrist flexion and extension. The proximal muscles were of normal power. In the lower limbs, the proximal muscles were MRC grade 5/5, bilaterally testing hip flexion, extension, hip adduction, abduction, knee flexion and extension. There was significant weakness and wasting of the tibialis anterior and gastrocnemius muscles bilaterally and MRC grade 1-2/5 testing foot dorsiflexion, eversion, inversion and plantar flexion. Sensory examination disclosed decreased sensation to pinprick and temperature, with intact proprioception in both upper and lower extremities. There was a significant decrease in vibration sense at both big toes. Deep tendon reflexes were 1+ testing the biceps, triceps and brachioradialis with trace at the patellae and no reflexes obtained at the ankles. He had flexor plantar responses. Tests of the cerebellum disclosed no abnormalities. His gait demonstrated a bilateral foot drop, and the Romberg test was positive.

An electromyography (EMG) study showed absence of the evoked sensory nerve action potentials (SNAPs) in either sural or superficial peroneal nerves. Evoked SNAPs showed reduced response amplitudes with normal conduction velocities in the median, ulnar and radial nerves. Evoked compound muscle action potentials showed reduced response amplitudes recording the APB and ADM muscles in the median and ulnar nerves, respectively, with normal conduction velocities. In both peroneal and tibial nerves, the presence of markedly reduced response amplitudes, prolonged distal latencies and moderate slowing of the 
motor conduction velocities (30-35 m/s) was noted. Needle EMG showed the presence of 1+ abnormal spontaneous activity in the form of positive sharp waves in testing the APB and ADM muscles, but not elsewhere, in the arm muscles sampled. The presence of highamplitude motor units and a reduced interference pattern with maximal effort was also noted in these small hand muscles. In the legs, ongoing abnormal spontaneous activity in the form of positive sharp waves $(2-3+)$ and fibrillation potentials was noted in a gradient most marked in the distal muscles (tibialis anterior, medial gastrocnemius and peroneus longus). In these distal muscles, motor unit analysis showed the presence of high-amplitude polyphasic units and a reduced interference pattern with maximal effort. Overall, the study confirmed the presence of a length-dependent sensory-motor polyneuropathy with acute and chronic axonal features which were more severe in the legs.

\section{Family Study}

Informed consent was obtained from the patient and members of his family. All members of this family are of Polish descent, and there is no consanguinity. The patient has two siblings (fig. 1). The patient's father, aged 62 years, and his sister, aged 28 years, had no symptoms suggestive of neuropathy and normal neurological examinations.

His mother, aged 59 years, complained of imbalance and mild numbness restricted to her feet. The exact onset of these symptoms was not clear but may have developed over the last 10 years prior to this evaluation. Her neurological examination showed a normal mental status, and cranial nerve examination was normal too. Her stretch reflexes were normoactive at the biceps, triceps and brachioradialis and mildly reduced at the patella. Her ankle jerks could not be elicited, and she had flexor plantar reflexes. Sensory examination revealed a decrease in pin prick, vibration and proprioception distally in her legs. Power testing was normal except she could not walk on her toes or heels. She had gait imbalance, could not perform a tandem walk and had a positive Romberg test. An EMG was performed and showed normal compound muscle action potentials and SNAPs in the right median and ulnar nerves. Evoked SNAPs were not obtained in either sural or superficial peroneal nerves. In both peroneal and tibial nerves, the motor studies showed markedly reduced response amplitudes with moderate slowing of the motor conduction velocities $(30-35 \mathrm{~m} / \mathrm{s})$. A concentric needle EMG showed abnormal spontaneous activity in the form of positive sharp waves $(2-3+)$ and fibrillation potentials, with the changes most prominent in the distal leg muscle samples. In these distal leg muscles, high-amplitude polyphasic motor units were noted, and the interference pattern was reduced with maximal effort. No abnormalities were noted in sampling the arm muscles. Overall, the study confirmed the presence of a sensory motor axonal length-dependent polyneuropathy with acute and chronic neurogenic changes.

His brother, aged 37 years, was not available for examination, but a history of his clinical status was obtained independently from two family members. He had complaints of numbness in his feet and imbalance beginning in his mid-twenties, which slowly progressed over the next 10 years. At present, he is still ambulatory without the need for any assistive devices and has no complaints of weakness or numbness in hands.

\section{Genetic Analysis}

A genetic study was performed to analyze the genotype-phenotype relationship in each individual of the family. Following Institutional Review Board-approved policies and procedures, blood samples of the affected patient, parents and siblings were obtained and DNA was extracted. Whole-exome sequencing of the patient was performed by a commercial sequencing company. Exome capture was performed by HiSeq2000 using a paired-end $(2 \times$ 


\section{Case Reports in Neurology}

100) protocol, Illumina raw data processing and an Agilent SureSelect exome kit for exome enrichment. The sequences were aligned to human genome reference (GRch37 version). Nucleotide-level variation analysis of the exome sequence data was performed using various tools available on the DNA nexus platform (https://dnanexus.com/). The variants obtained were further annotated using the Ensembl variant effect predictor tool (Ensembl release 75, February 2014; http://useast.ensembl.org/info/docs/tools/vep/index.html). These results were further filtered using SIFT and Polyphen, and variants with deleterious, possible damaging and unknown effects were selected. Single nucleotide polymorphisms involving genes known to cause CMT were then analyzed.

\section{Results}

Two heterozygous missense variants were identified: the previously described variant rs121909079 on chromosome 3 at position 128526470; this c.484G >A results in a p.V162M change in the $R A B 7 A$ gene [2]. A second heterozygous $\mathrm{C} / \mathrm{G}$ variant was detected on chromosome 9 at position 130219669; this c.643C $>$ G change results in a p.P215A change in the LRSAM1 gene. These single nucleotide polymorphisms were analyzed in all the members of the family and reconfirmed by polymerase chain reaction amplification and Sanger's resequencing (fig. 1). This LRSAM1 variant is novel, and protein modeling using SIFT and Polyphen analysis shows that it is likely a damaging mutation.

\section{Discussion}

All disorders classified as CMT2 have similar clinical and electrophysiological presentations and are further categorized by molecular genetic analysis. CMT2B is caused by mutations in the RAB7A gene which encodes Rab7, a small, RAS-related GTP-binding protein that regulates vesicular transport, biogenesis and the maturation of lysosomes and autophagosomes in the endocytic pathway [4-6]. It is also involved in the retrograde axonal transport of neurotrophin receptors and the formation of multivesicular bodies involved in the degradation of these receptors [7-9]. The mutation in the RAB7A gene found in our patient has been previously described in two families, one of American descent and another of Scottish origin [2]. There are phenotypic similarities between these two previously reported families, with both of them exhibiting mutilating neuropathic ulcerations as a prominent clinical feature. In the American family, the larger of the two, with 10 affected individuals, the age of onset ranged from late teens to early thirties, and all had foot sores or mutilating foot ulcerations [10]. In the second family, with 5 affected members in 3 generations, a relatively mildly affected woman had a son who was severely affected with mutilating foot ulcers raising the issue of gender-related gene penetrance [11].

CMT2P is caused by mutations in the LRSAM1 gene that encodes an ubiquitin-protein ligase which regulates the Tumor Susceptibility 101 (Tsg101)-associated complex responsible for sorting internalized cell-surface receptor proteins into cytoplasm-containing vesicles [12]. LRSAM1 protein is also involved in the regulation of signaling pathways, cell adhesion and the mediation of self-ubiquitylation following receptor endocytosis which promotes their proteosomal degradation [13]. Interestingly, mutations in this gene can cause both AR and $\mathrm{AD}$ forms depending upon the nature of the mutation. Specific dominant mutations are hypothesized to cause a gain rather than a loss of function as would be expected with those 
following an AR pattern. The LRSAM1 mutation found in this family is recessive since the father and daughter carry the mutation and have no symptoms or signs of neuropathy.

Epidermal growth factor receptor (EGFR) signaling is reported to play a role in the maintenance of glia, the trafficking of the endosome to the lysosome and in autophagy. In adult neurons, RAB7A and TSG101 genes affect the distribution of EGFR, the regulation of the recycling and transport of the EGF: EGFR complex and their lysosomal degradation $[14,15]$. Mutations in RAB7A and LRSAM1 genes lead to common pathogenic mechanisms resulting in impaired trafficking of the endosome to the lysosome and the accumulation of the inactive EGFR in endosomal vesicles. This in turn results in the accumulation of multivesicular bodies and the eventual loss of EGFR recycling (fig. 2). The overall impact of impaired EGFR signaling is a possible acceleration of neuronal degeneration, which can clinically manifest as a more severe phenotype. In this family, even though both brothers inherited the same RAB7A mutation, the individual carrying an additional mutation in the LRSAM1 gene became symptomatic 10 years earlier and is more severely affected. Given that both of these mutations occur in genes encoding proteins involved in a cellular trafficking pathway, we propose that the interaction between the mutant RAB7A and LRSAM1 genes contribute to the more severe phenotype. Perhaps in this individual, the presence of the LRSAM1 mutation makes him more vulnerable to the effects of the mutation in the $R A B 7 A$ gene.

The study of our family confirms and extends the phenotypic variability described in reports of patients with CMT2B. Unlike the descriptions of these previous families, no member of our family has had ulceromutilation as a clinical feature. In this family, the age of onset in the affected mother is much later than that of either of her sons, confirming the previous observation that this $R A B 7 A$ disease mutation may be less penetrant in woman compared with men [2]. There is no research exploring any potential relationship that could explain this phenomenon. However, phenotypic variability is a well-recognized clinical feature and has been observed even in Mendelian disorders. Synergistic interaction between heterozygous mutations in RAB7A and LRSAM1 genes could account for the phenotypic variability observed in the affected brothers.

\section{Statement of Ethics}

Informed consent was obtained from all members of this family, and the study was conducted following local Institutional Review Board-approved policies and procedures.

\section{Disclosure Statement}

The authors declare that there is no conflict of interest regarding the publication of this paper.

\section{References}


-3 Guernsey DL, Jiang H, Bedard K, Evans SC, Ferguson M, Matsuoka M, Macgillivray C, Nightingale M, Perry S, Rideout AL, Orr A, Ludman M, Skidmore DL, Benstead T, Samuels ME: Mutation in the gene encoding ubiquitin ligase LRSAM1 in patients with Charcot-Marie-Tooth disease. PLoS Genet 2010;6:e1001081. DOI: 10.1371/journal.pgen.1001081.

-4 Feng Y, Press B, Wandinger-Ness A: Rab7: an important regulator of late endocytic membrane traffic. Cell Biol 1995;131:1435-1452.

5 Bucci C, Thomsen P, Nicoziani P, McCarthy J, van Deurs B: Rab7: a key to lysosome biogenesis. Mol Biol Cell 2000;11:467-480.

-6 Jäger S, Bucci C, Tanida I, Ueno T, Kominami E, Saftig P, Eskelinen EL: Role for Rab7 in maturation of late autophagic vacuoles. J Cell Sci 2004;117:4837-4848.

-7 Saxena S, Bucci C, Weis J, Kruttgen A: The small GTPase Rab7 controls the endosomal trafficking and neuritogenic signaling of the nerve growth factor receptor TrkA. J Neurosci 2005;25:10930-10940.

8 Deinhardt K, Salinas S, Verastegui C, Watson R, Worth D, Hanrahan S, Bucci C, Schiavo G: Rab5 and Rab7 control endocytic sorting along the axonal retrograde transport pathway. Neuron 2006;52:293-305.

9 Progida C, Malerød L, Stuffers S, Brech A, Bucci C, Stenmark H: RILP is required for the proper morphology and function of late endosomes. J Cell Sci 2007;120:3729-3737.

10 Kwon JM, Elliott JL, Yee WC, Ivanovich J, Scavarda NJ, Moolsintong PJ, Goodfellow PJ: Assignment of a second Charcot-Marie-Tooth type II locus to chromosome 3q. Am J Hum Genet 1995;57:853-858.

11 De Jonghe P, Timmerman V, FitzPatrick D, Spoelders P, Martin JJ, Van Broeckhoven C: Mutilating neuropathic ulcerations in a chromosome 3q13-q22 linked Charcot-Marie-Tooth disease type 2B family. J Neurol Neurosurg Psychiatry 1997;62:570-573.

12 Amit I, Yakir L, Katz M, Zwang Y, Marmor MD, Citri A, Shtiegman K, Alroy I, Tuvia S, Reiss Y, Roubini E, Cohen M, Wides R, Bacharach E, Schubert U, Yarden Y: Tal, a Tsg101-specific E3 ubiquitin ligase, regulates receptor endocytosis and retrovirus budding. Genes Dev 2004;18:1737-1752.

13 Bogdanik LP, Sleigh JN, Tian C, Samuels ME, Bedard K, Seburn KL, Burgess RW: Loss of the E3 ubiquitin ligase LRSAM1 sensitizes peripheral axons to degeneration in a mouse model of Charcot-Marie-Tooth disease. Dis Model Mech 2013;6:780-792.

14 Razi M, Futter CE: Distinct roles for Tsg101 and Hrs in multivesicular body formation and inward vesiculation. Mol Biol Cell 2006;17:3469-3483.

15 Rush JS, Ceresa BP: RAB7 and TSG101 are required for the constitutive recycling of unliganded EGFRs via distinct mechanisms. Mol Cell Endocrinol 2013;381:188-197. 


\section{Case Reports in \\ Neurology}

Peddareddygari et al.: Factors Affecting Phenotype Variability in a Family with CMT2B: Gender and LRSAM1 Genotype

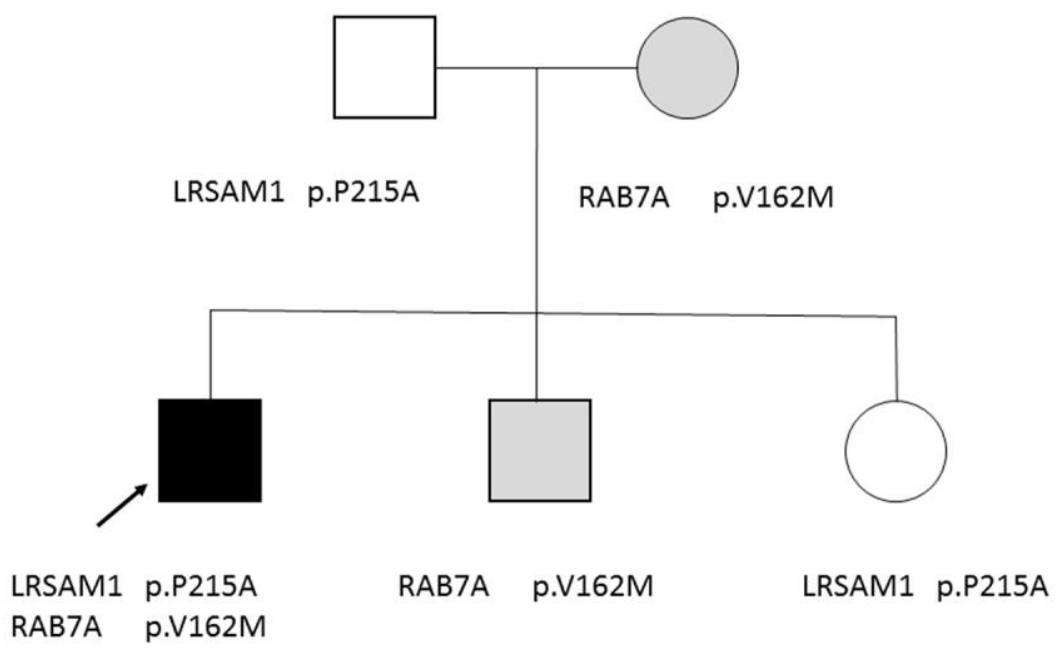

Fig. 1. Pedigree of the family showing individuals with variable clinical presentation and their inherited mutations. Squares indicate males and circles females. Black indicates the affected individual with severe clinical presentation, grey indicates mild clinical features, and white indicates individuals without symptoms.

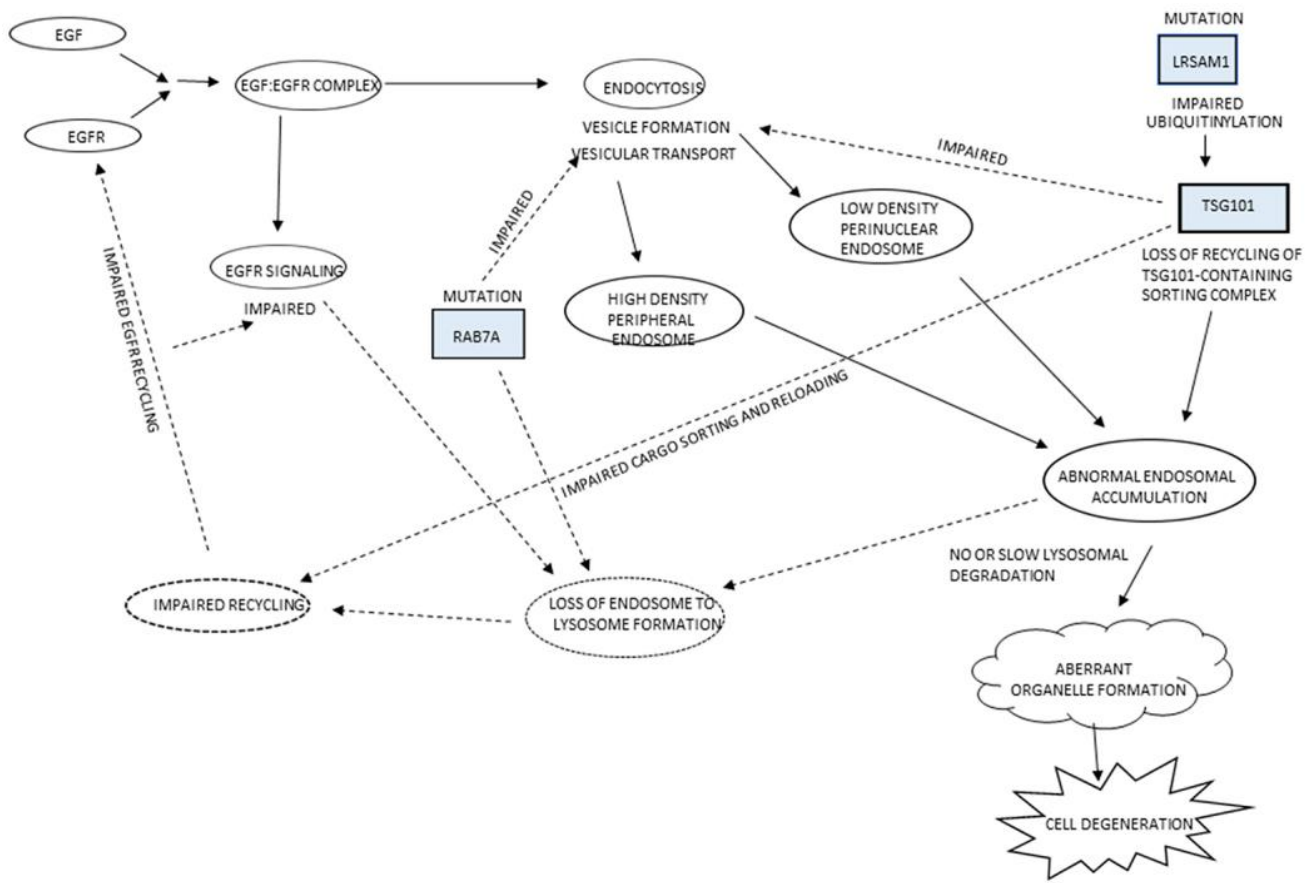

Fig. 2. Impaired transport and recycling of EGFR resulting from the synergistic interaction between mutations in LRSAM1 and RAB7A genes leading to altered endosomal and nuclear signaling, aberrant organelle formation and cell degeneration. 